\title{
SPAG9 promotes the migration and invasion of melanoma cells by regulating the MAPK pathway
}

\author{
Yongjun Liu ${ }^{1}$, Xuefeng Zhu ${ }^{1}$, Xiaoen You ${ }^{1}$, Runfang Kang ${ }^{2 *}$ \\ ${ }^{1}$ Department of Cosmetic and Plastic Surgery Burn Wound Repair, Lishui City People's Hospital, '2Department of Dermatology, \\ Lishui Second People's Hospital, Lishui, Zhejiang Province 323000, China \\ *For correspondence: Email: kangrunfang10317@163.com; Tel: +86-0578-2298812
}

\begin{abstract}
Purpose: To determine the regulatory role of sperm-associated antigen 9 (SPAG9) in melanoma cell growth.

Methods: Immunohistochemical analysis was performed to investigate SPAG9 expression in melanoma tissues. Western blot was used to evaluate SPAG9 expression in melanoma tissues and cells. Melanoma cells were transfected with an siRNA targeting SPAG9 or a SPAG9 overexpression vector. Cell migration and invasion were examined by wound healing and transwell assays. The effects of SPAG9 on the epithelial-mesenchymal transition and mitogen-activated protein kinase (MAPK) pathway in melanoma cells were assessed by western blot.

Results: SPAG9 expression was enhanced in melanoma tissues and cells. SiRNA-mediated silencing of SPAG9 repressed melanoma cell migration and invasion, and SPAG9 overexpression contributed to melanoma cell metastasis. In melanoma cells transfected with siRNA-SPAG9, E-cadherin expression decreased while vimentin and matrix metalloproteinase (MMP)-2/9 expression increased. However, ectopic expression of SPAG9 reversed this expression of E-cadherin, vimentin, and MMP-2/9. Silencing of SPAG9 decreased the phosphorylation of MAPKs, including p-p38, p-ERK and p-JNK, in melanoma cells. SPAG9 overexpression upregulated phosphorylation of MAPKs.

Conclusion: SPAG9 promotes the migration and invasion of melanoma cells by activating the MAPK pathway.
\end{abstract}

Keywords: SPAG9, Cell migration, Cell invasion, Melanoma, MAPKs, E-cadherin, Vimentin, Matrix metalloproteinase (MMP)

\begin{abstract}
This is an Open Access article that uses a fund-ing model which does not charge readers or their institutions for access and distributed under the terms of the Creative Commons Attribution License (http://creativecommons.org/licenses/by/4.0) and the Budapest Open Access Initiative (http://www.budapestopenaccessinitiative.org/read), which permit unrestricted use, distribution, and reproduction in any medium, provided the original work is properly credited.
\end{abstract}

Tropical Journal of Pharmaceutical Research is indexed by Science Citation Index (SciSearch), Scopus, International Pharmaceutical Abstract, Chemical Abstracts, Embase, Index Copernicus, EBSCO, African Index Medicus, JournalSeek, Journal Citation Reports/Science Edition, Directory of Open Access Journals (DOAJ), African Journal Online, Bioline International, Open-J-Gate and Pharmacy Abstracts

\section{INTRODUCTION}

Melanoma is a highly aggressive cancer that is caused by exposure to ultraviolet radiation [1,2]. Melanomas tend to metastasize into other organs, including the liver, lung, brain, and bone [3]. The 10-year survival rate for metastatic melanoma is only $10-15 \%$ [4]; therefore, novel, effective, and safe therapeutic strategies are urgently needed to improve the diagnosis and prognosis of melanoma.

Activation of mitogen-activated protein kinases (MAPKs) has been shown to contribute to the epithelial-mesenchymal transformation and extravasation of tumor cells and to promote 
tumor cell migration and invasion capabilities [5]. An oncogenic MAPK pathway has been shown to be activated in melanoma cells [6], and blocking this MAPK pathway suppressed cell proliferation and invasion of melanoma [8]. Therefore, MAPKs have potential as therapeutic targets for the treatment of melanoma.

Sperm-associated antigen 9 (SPAG9), a cancer/testis antigen, was found to be expressed abnormally in various human malignancies and to play a key role in tumor proliferation and invasion [9]. For example, overexpression of SPAG9 related positively with metastasis of hepatocarcinoma [10], and ectopic expression of SPAG9 in prostate cancer cells promoted cell proliferation, metastasis, and angiogenesis [11]. SPAG9 was also implicated in the metastasis of osteosarcoma [12]. Silencing of SPAG9 retarded triple-negative breast cancer cell growth and metastasis [13]. Previous studies have shown that SPAG9 binds to c-Jun $\mathrm{N}$-terminal kinases (JNKs) [14] and plays an important regulatory role in several physiologic processes, such as cell survival and tumor development [15]. Therefore, this study investigated the hypothesis that SPAG9 contributes to melanoma cell metastasis through activation of MAPKs.

\section{METHODS}

\section{Human specimens and immunohistochemical analysis}

Forty patients with melanoma were recruited from Lishui City People's Hospital. Melanoma and adjacent normal tissues were collected from patients who signed informed consent forms. This study was approved by the Ethics Committee of Lishui City People's Hospital (approval no. LLW-FO-403) and performed in accordance with the 1964 Helsinki Declaration and its later amendments for ethical research involving human subjects [16].

Formalin-fixed and paraffin-embedded melanoma tissues were cut into $4 \mu \mathrm{m}$ thick sections. The sections were dewaxed, rehydrated, and endogenous peroxidase was blocked by treating the sections with $3 \% \mathrm{H}_{2} \mathrm{O}_{2}$. After immersion in Tris-EDTA buffer for antigen retrieval, sections were incubated with $0.3 \%$ goat serum and $4 \%$ dry milk, incubated with a specific anti-SPAG9 antibody (1:100; Abcam, Cambridge, MA, USA), incubated with horseradish peroxidase-labeled anti-rabbit secondary antibody (Abcam), and then counterstained with hematoxylin before being viewed under the microscope (Olympus, Tokyo, Japan).

\section{Cell culture and transfection}

Melanoma cell lines A375, G361, SK-MEL-1, and A2058 and human epidermal keratinocyte line HACAT were acquired from Ningbo Mingzhou Biotechnology Company (Mingzhoubio, China). Cells were cultured in RPMI-1640 medium (Life Technologies, Carlsbad, CA, USA) containing $10 \%$ fetal bovine serum (Beyotime, Beijing, China).

SiRNAs to silence SPAG9 (si-SPAG9) and a control (si-NC) were synthesized by Genepharma (Suzhou, China). Full length SPAG9 was inserted into the pcDNA vector (Invitrogen, Carlsbad, CA, USA) to overexpress SPAG9. A375 cells were cultured in sterile 6-well plates for $24 \mathrm{~h}$ before transfection with siSPAG9, si-NC, pcDNA, or pcDNA-SPAG9 using Lipofectamine 2000 (Invitrogen).

\section{Wound healing and transwell assays}

Two days post-transfection, a pipette was used to scratch the middle of each well, and plates were incubated for another $24 \mathrm{~h}$ before the widths of the scratches were measured under the microscope. For the transwell assay, transfected A375 cells in $200 \mu \mathrm{L}$ of RPMI- 1640 medium were seeded into the upper chamber of Matrigel precoated wells (Corning, Tewksbury, MA, USA). To the lower chamber, $400 \mu \mathrm{L}$ of RPMI-1640 medium containing $20 \%$ fetal bovine serum was added. After $48 \mathrm{~h}$, the cells that invaded the lower chamber were fixed with methanol, stained with crystal violet, and counted by microscopy.

\section{Western blot}

Melanoma tissues or A375 cells were lysed in radioimmunoprecipitation assay lysis buffer (Beyotime Institute of Biotechnology, Haimen, China), and the protein concentrations of the cell lysates were determined using the Bicinchoninic Acid protein assay kit (Beyotime Institute of Biotechnology). Lysates $(30 \mu \mathrm{g})$ were separated by sodium dodecyl sulfate polyacrylamide gel electrophoresis and then transferred to polyvinylidene fluoride membranes. The membranes were blocked with $5 \%$ milk and then probed at $4^{\circ} \mathrm{C}$ overnight with the following primary antibodies: anti-SPAG9, anti-E-cadherin, and anti-vimentin (1:2500, Bioworld Technology, St. Louis Park, MN, USA); anti-MMP2, antiMMP9, and anti-GAPDH (1:3500, Bioworld Technology); and anti-p38, anti-p-p38, anti-ERK, anti-p-ERK, anti-JNK, and anti-p-JNK (1:4500, Bioworld Technology). The membranes were then incubated with horseradish peroxidaselabeled goat anti-rabbit IgG (1:5000, Bioworld 
Technology) for $2 \mathrm{~h}$, and immunoreactivity was assessed using enhanced chemiluminescence detection reagent (Beyotime Institute of Biotechnology) and ImageJ software.

\section{Statistical analysis}

Data were expressed as mean \pm standard error of the mean (SEM) and were analyzed by oneway analysis of variance or the student's t test using GraphPad Prism software (5). $P<0.05$ was considered statistically significant.

\section{RESULTS}

\section{Enhanced SPAG9 expression in melanoma tissues and cell lines}

To investigate the role of SPAG9 in melanoma, SPAG9 expression in melanoma tissues was assessed by immunohistochemistry and western blot analysis, and higher SPAG9 expression was found in melanoma tissues than in adjacent normal tissues (Figure 1A, B). SPAG9 expression was also upregulated in the melanoma cell lines A375, G361, SK-MEL-1, and A2058, compared to the human epidermal keratinocyte line HACAT (Figure 1C), suggesting that SPAG9 may participate in melanoma progression.

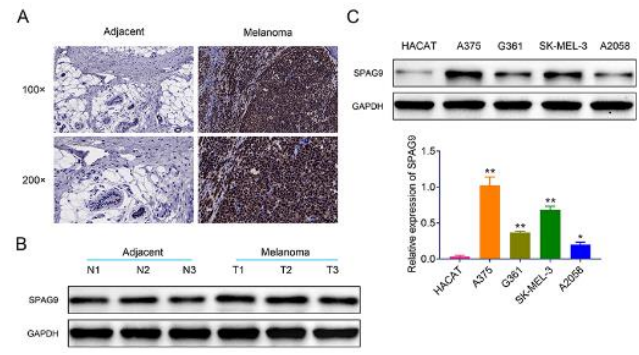

Figure 1. Enhanced SPAG9 expression in melanoma. (A) Immunohistochemical analysis showed that SPAG9 expression was upregulated in melanoma tissues when compared with adjacent normal tissues. (B) Western blot analysis showed that SPAG9 was upregulated in melanoma tissues compared to adjacent normal tissues. (C) Western blot analysis showed that SPAG9 was upregulated in melanoma cell lines A375, G361, SK-MEL-1, and A2058 compared to human epidermal keratinocyte line HACAT. vs. HACAT, ${ }^{*} P<0.05,{ }^{* *} p<0.01$

\section{SPAG9 contributes to migration and invasion of melanoma cells}

A375 cells were transfected with si-SPAG9 or pcDNA-SPAG9 to evaluate whether SPAG9 contributes to melanoma progression. Cells transfected with si-SPAG9 expressed less SPAG9 protein than cells transfected with siNC
(Figure 2A), and cells transfected with pcDNASPAG9 expressed more SPAG9 protein than cells transfected with pcDNA (Figure 2A). Functional assays showed that ectopic expression of SPAG9 in A375 cells promoted cell migration (Figure 2B, D) and invasion (Figure 2C, $E)$, whereas silencing of SPAG9 in A375 cells suppressed cell migration (Figure 2B, D) and invasion (Figure 2C, E). These results demonstrated the oncogenic role of SPAG9 in melanoma.
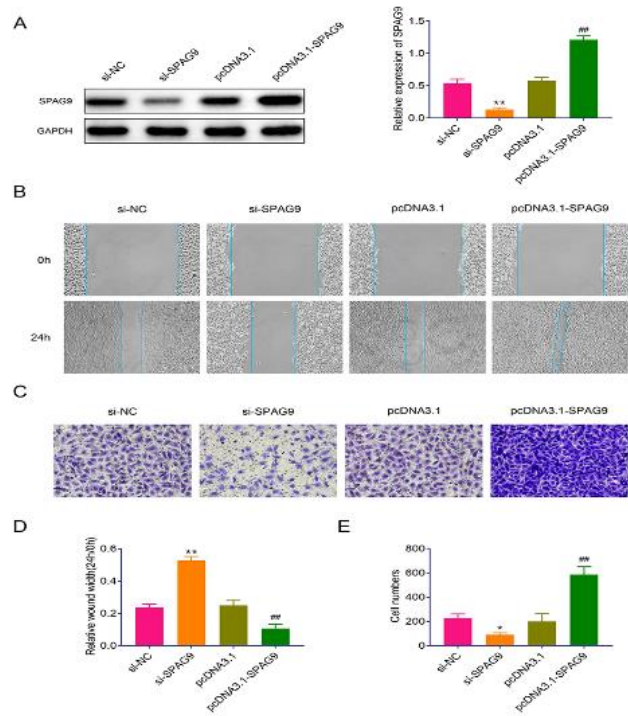

Figure 2. SPAG9 contributes to migration and invasion of melanoma cells. (A) A375 cells transfected with si-SPAG9 had lower SPAG9 protein expression than cells transfected with si-NC, and A375 cells transfected with pcDNA-SPAG9 had higher SPAG9 protein expression than cells transfected with the pcDNA vector alone. (B) Ectopic expression of SPAG9 promoted migration of $\mathrm{A} 375$ cells, and silencing of SPAG9 suppressed cell migration. (C) Ectopic expression of SPAG9 promoted invasion of A375 cells, and silencing of SPAG9 suppressed invasion. (D) Wound widths were measured after $24 \mathrm{~h}$. Ectopic expression of SPAG9 decreased the wound width, and silencing of SPAG9 decreased the wound width. (E) Quantification of cell invasion showed that ectopic expression of SPAG9 increased the number of invasive cells, and silencing of SPAG9 decreased the number of invasive cells. ${ }^{*},{ }^{* *}$ si-SPAG9 vs. siNC, $p<$ $0.05, p<0.01$. \#\# pcDNA3.1-SPAG9 vs. pcDNA3.1, $p$ $<0.01$

SPAG9 contributes to the epithelialmesenchymal transformation of melanoma cells

In addition to the effect on melanoma cell migration and invasion, the effect of SPAG9 on the epithelial-mesenchymal transformation was assessed. In A375 cells transfected with siSPAG9, E-cadherin protein expression 
increased, whereas vimentin, matrix metalloproteinase 2 (MMP-2), and MMP-9 protein expression decreased (Figure 3). Moreover, in A375 cells overexpressing SPAG9, E-cadherin protein expression decreased, whereas vimentin, MMP-2, and MMP-9 protein expression increased (Figure 3). These results show that SPAG9 contributes to melanoma cell metastasis.

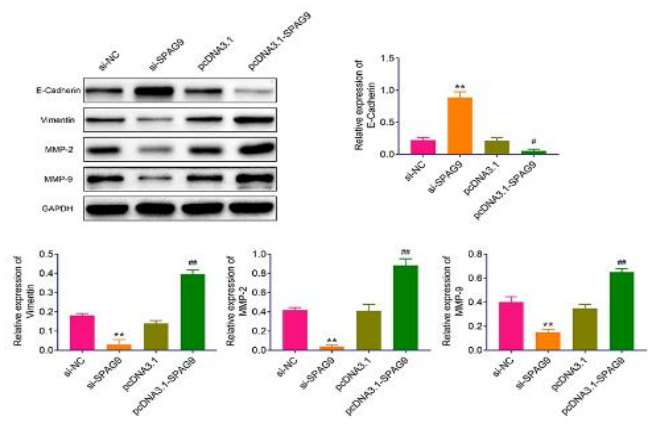

Figure 3. SPAG9 contributes to the epithelialmesenchymal transformation of melanoma cells. Ectopic expression of SPAG9 reduced E-cadherin protein expression and increased vimentin, MMP-2, and MMP-9 protein expression in A375 cells. Silencing of SPAG9 reversed these protein expression results. ** si-SPAG9 vs. siNC, $p<0.01$. \#, \#\# pcDNA3.1SPAG9 vs. pcDNA3.1, $p<0.05, p<0.01$

SPAG9 promotes activation of MAPKs in melanoma

Transfection with si-SPAG9 or pcDNA-SPAG9 had no significant effect on protein expression of the MAPKs p38, ERK, and JNK (Figure 4). However, phosphorylation of p38, ERK, and JNK was downregulated by silencing of SPAG9 (Figure 4) and upregulated by overexpression of SPAG9 (Figure 4). These results suggest that SPAG9 promotes activation of MAPKs in melanoma to promote cell metastasis.

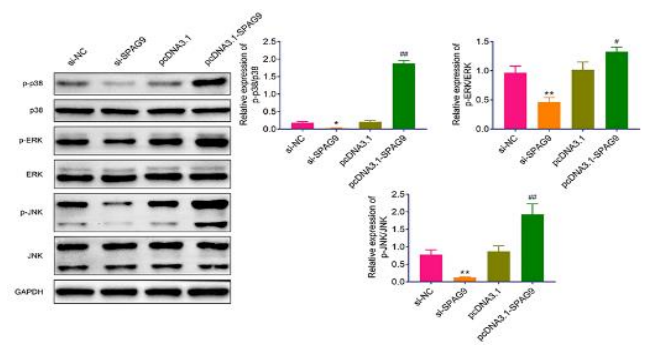

Figure 4. SPAG9 promotes activation of MAPKs in melanoma. Ectopic expression of SPAG9 in A375 cells increased protein expression of p-p38, p-JNK, and $p$-ERK, whereas silencing of SPAG9 reduced $p$ p38, p-JNK, and p-ERK protein expression. ${ }^{*},{ }^{* *}$ siSPAG9 vs. siNC, $p<0.05, p<0.01$. \#, \#\# pcDNA3.1SPAG9 vs. pcDNA3.1, $p<0.05, p<0.01$

\section{DISCUSSION}

Cancer/testis antigens, including SPAGs, are widely considered therapeutic targets for the prevention of tumors [17], especially melanoma [18]. Comprehensive expression pattern analyses of SPAGs in various cancerous tissues showed that SPAGs have potential as immunotherapeutic targets [19]. Because SPAG9 was shown to be dysregulated in various tumors and to induce an immune response, it has been suggested as a potential target for cancer therapy [9]. In this study, the functional role of SPAG9 in melanoma cell growth and metastasis was investigated.

In this study, we found that SPAG9 expression is upregulated in melanoma tissues and cells. In addition, statistical analysis has shown that SPAG9 expression is associated with breast cancer tumor stage, size, and lymph node metastasis [20]. These data indicate that the diagnostic and/or prognostic roles of SPAG9 in melanoma should be investigated further by analyzing the relationship between SPAG9 expression and the clinicopathologic features of melanoma patients. In this study, functional assays showed that overexpression of SPAG9 promoted melanoma cell migration and invasion and that silencing of SPAG9 suppressed tumor cell metastasis indicating that SPAG9 plays an oncogenic role in melanoma. In addition, a previous study implicated that SPAG9 plays a role in the cell cycle progression of tumor cells [9], thus the effect of SPAG9 on melanoma cell proliferation and apoptosis should be investigated.

The epithelial-mesenchymal transformation, the loss of epithelial character and the gain of mesenchymal morphology, is critical for promoting cancer progression by inducing melanoma cell migration and invasion [21]. Downregulation of the epithelial protein $\mathrm{E}$ cadherin and upregulation of mesenchymal proteins, including $\mathrm{N}$-cadherin and vimentin, are markers of the epithelial-mesenchymal transformation [22]. In this study, overexpression of SPAG9 reduced E-cadherin protein expression and increased vimentin protein expression in melanoma cells, thus promoting the epithelial-mesenchymal transformation. Moreover, MMPs, such as MMP-2 and MMP-9, play key roles in the proteolytic degradation of the extracellular matrix surrounding primary tumor tissues, which is required for cancer cell migration and invasion [11]. SPAG9 was shown to promote prostate cancer cell migration and invasion through increases in MMP-2 and MMP9 expression [11]. In this study, silencing of 
SPAG9 reduced MMP-2 and MMP-9 expression and repressed melanoma cell metastasis.

The MAPK pathway is an oncogenic signaling pathway in melanoma patients; phosphorylation of MAPKs promote tumor development and inhibitors of MAPKs have led to major advances in melanoma prevention [23]. SPAG9 functions as a scaffolding protein to modulate the MAPK pathway through interaction with JNKs, thus SPAG9 participates in tumor growth and metastasis [9]. SPAG9 promoted phosphorylation of p38, JNK, and ERK and enhanced proliferation and metastasis of prostate cancer [24]. The SPAG9/p38 axis was validated as a potential target for the treatment of liver cancer [25]. In this study, protein expression of $\mathrm{p}-\mathrm{p} 38, \mathrm{p}-\mathrm{JNK}$, and $\mathrm{p}$-ERK were reduced by silencing of SPAG9 and enhanced by overexpression of SPAG9 in melanoma cells indicating that the MAPK pathway is involved in SPAG9-mediated melanoma cell metastasis.

\section{CONCLUSION}

SPAG9 promotes tumor metastasis and the epithelial-mesenchymal transformation through activation of the MAPK pathway. Thus, silencing of SPAG9 may be a novel strategy for the management of melanoma, but this needs to be further investigated in an in vivo xenograft tumor model.

\section{DECLARATIONS}

\section{Acknowledgement}

Thanks are due to Mr. Amjad Farooq for his technical assistance, and BioMES, Alpha Genomics Private Limited, Islamabad - Pakistan for editing and reviewing the manuscript. Thanks are also due to the patients who participated in the study.

\section{Conflict of interest}

No conflicts of interest to disclose regarding this work.

\section{Availability of data and materials}

All data generated or analyzed during this study are included in this published article.

\section{Contribution of authors}

We declare that this work was done by the authors named in this article and all liabilities pertaining to claims relating to the content of this article will be borne by the authors. Yongjun Liu and Xuefeng Zhu designed the study and supervised the data collection. Xiaoen You analyzed and interpreted the data. Runfang Kang prepared the manuscript for publication and reviewed the draft of the manuscript. All authors read and approved the manuscript.

\section{Open Access}

This is an Open Access article that uses a funding model which does not charge readers or their institutions for access and distributed under the terms of the Creative Commons Attribution License (http://creativecommons.org/licenses/by/ 4.0) and the Budapest Open Access Initiative (http://www.budapestopenaccessinitiative.org/rea d), which permit unrestricted use, distribution, and reproduction in any medium, provided the original work is properly credited.

\section{REFERENCES}

1. Viceconte $N$, Dheur M-S, Majerova E, Pierreux CE, Baurain J-F, van Baren $N$, Decottignies $A$. Highly Aggressive Metastatic Melanoma Cells Unable to Maintain Telomere Length. Cell Rep 2017; 19(12): 2529-2543.

2. Sun $X$, Yang $P$, Jiang $Y$. CD147 promotes melanoma cell growth via SOX4-mediated glycolytic metabolism. Trop J Pharm Res 2020; 19(12): 2521-2527.

3. Carr S, Smith C, Wernberg J. Epidemiology and Risk Factors of Melanoma. Surg Clin N Am 2020; 100(1): 112.

4. O'Neill CH, Scoggins CR. Melanoma. J Surg Oncol 2019; 120(5): 873-881.

5. Ivan $d B B$, Nebreda $A$. Roles of p38 MAPKs in invasion and metastasis. Biochem Soc T 2012; 40: 79-84.

6. Shoushtari AN, Chatila WK, Arora A, Sanchez-Vega F, Kantheti HS, Rojas Zamalloa JA, Krieger $P$, Callahan MK, Betof Warner A, Postow MA et al. Therapeutic Implications of Detecting MAPK-Activating Alterations in Cutaneous and Unknown Primary Melanomas. Clin Cancer Res 2021.

7. Amaral T, Sinnberg T, Meier F, Krepler C, Levesque M, Niessner $H$, Garbe $C$. The mitogen-activated protein kinase pathway in melanoma part I - Activation and primary resistance mechanisms to BRAF inhibition. Eur J Cancer 2017; 73: 85-92.

8. Pathria G, Verma S, Yin J, Scott DA, Ronai ZeA. MAPK signaling regulates $c-M Y C$ for melanoma cell adaptation to asparagine restriction. EMBO Rep 2021; 22(3): e51436.

9. Pan J, Yu H, Guo Z, Liu Q, Ding M, Xu K, Mao L. Emerging role of sperm-associated antigen 9 in tumorigenesis. Biomed Pharmacother 2018; 103: 12121216. 
10. Yan Q, Lou G, Qian Y, Qin B, Xu X, Wang Y, Liu Y, Dong $X$. SPAG9 is involved in hepatocarcinoma cell migration and invasion via modulation of ELK1 expression. Oncotargets Ther 2016; 9: 1067-1075.

11. Chen F, Lu Z, Deng J, Han X, Bai JIN, Liu Q, Xi Y, Zheng J. SPAG9 expression is increased in human prostate cancer and promotes cell motility, invasion and angiogenesis in vitro. Oncol Rep 2014; 32(6): 25332540.

12. Yang $X$, Zhou W, Liu S. SPAG9 controls the cell motility, invasion and angiogenesis of human osteosarcoma cells. Exp Ther Med 2016; 11(2): 637-644.

13. Sinha A, Agarwal S, Parashar D, Verma A, Saini S, Jagadish $N$, Ansari AS, Lohiya NK, Suri A. Down regulation of SPAG9 reduces growth and invasive potential of triple-negative breast cancer cells: possible implications in targeted therapy. J Exp Clin Canc Res 2013; 32(1): 69-69.

14. Jagadish $N$, Rana $R$, Selvi R, Mishra D, Garg M, Yadav $S$, Herr JC, Okumura K, Hasegawa A, Koyama $K$ et al. Characterization of a novel human sperm-associated antigen 9 (SPAG9) having structural homology with cJun N-terminal kinase-interacting protein. Biochemical J 2005; 389(Pt 1): 73-82.

15. Rana $R$, Jagadish N, Garg $M$, Mishra $D$, Dahiya $N$, Chaurasiya $D$, Suri A. Small interference RNA-mediated knockdown of sperm associated antigen 9 having structural homology with c-Jun N-terminal kinaseinteracting protein. Biochem Bioph Res Co 2006; 340(1): 158-164.

16. World Medical A. World Medical Association Declaration of Helsinki: Ethical Principles for Medical Research Involving Human Subjects. JAMA 2013; 310(20): 21912194.

17. Salmaninejad A, Zamani MR, Pourvahedi M, Golchehre Z, Hosseini Bereshneh A, Rezaei N. Cancer/Testis
Antigens: Expression, Regulation, Tumor Invasion, and Use in Immunotherapy of Cancers. Immunol Invest 2016; 45(7): 619-640.

18. Sigalotti L, Covre A, Zabierowski S, Himes B, Colizzi F, Natali PG, Herlyn M, Maio M. Cancer testis antigens in human melanoma stem cells: expression, distribution, and methylation status. J Cell Physiol 2008; 215(2): 287-291.

19. Silina $K$, Zayakin $P$, Kalnina $Z$, Ivanova L, Meistere I, Endzelinš E, Abols A, Stengrevics A, Leja M, Ducena K et al. Sperm-associated Antigens as Targets for Cancer Immunotherapy: Expression Pattern and Humoral Immune Response in Cancer Patients. J Immunother 2011; 34(1).

20. Naraghi H, Mahjoubi F, Nafissi N. Sperm-Associated Antigen 9 as a Candidate Diagnostic and Prognostic Biomarker in Breast Cancer. Middle East $\mathrm{J}$ Cancer 2020; 11: 415-422.

21. Hodorogea A, Calinescu A, Antohe $M$, Balaban $M$, Nedelcu RI, Turcu G, Ion DA, Badarau IA, Popescu CM, Popescu $R$ et al. Epithelial-Mesenchymal Transition in Skin Cancers: A Review. Anal Cell Pathol 2019; 2019: 3851576.

22. Pearlman RL, Montes de Oca MK, Pal HC, Afaq F. Potential therapeutic targets of epithelial-mesenchymal transition in melanoma. Cancer Lett 2017; 391: 125-140.

23. Sullivan RJ, Flaherty K. MAP kinase signaling and inhibition in melanoma. Oncogene 2013; 32(19): 23732379.

24. Xiao C, Li M, Huang Q, Si-Tu J. SPAG9 promotes prostate cancer proliferation and metastasis via MAPK signaling pathway. Am J Transl Res 2019; 11(8): 52495260.

25. Luo S, Ren B, Zou G, Liu J, Chen W, Huang Y, Chen X, FU Y. SPAG9/MKK3/p38 axis is a novel therapeutic target for liver cancer. Oncol Rep 2019; 41. 